\title{
SPACECRAFT MISSION OPERATIONS - LESSONS LEARNED FROM AUTOMATION
}

\author{
Ave K. Kludze, Jr, NASA Langley Research Center, Hampton, Virgina
}

\section{Introduction}

This study was initially undertaken to understand how commonalties among the application of proven automation processes such as aircraft control, nuclear power generation, auto manufacturing etc. could be applied to spacecraft operations at NASA. These industries applied automation to reduce human repetitive task and mitigate risk, rather than create complete "lights out" operations as has been the goal at NASA.

In order to better understand the implementations, benefits, and cost savings of automation, a questionnaire was developed and sent out addressing the perception, implementation, approach, cost savings and human factors involved in automation. Of the 160 questionnaires sent out in July of 2000 , approximately $45 \%$ responses were received by the deadline. Some of the responses came from Flight Operation Teams (FOTs) members who have been on other automated projects and organizations internal and external to NASA. Several responses were also received from the automobile, nuclear, power, and airline industries. The following is a breakdown of the respondents:

- 160 questionnaires were sent out with 72 responses been received by the deadline.

- Out of the 72,45 of the respondents had a bachelor's degree or higher.

- Of the 72 responses: 33 came from flight operations, 10 from pilots of 3 major airlines, 12 from the power industry, 14 from the automobile industry, and 3 from other groups

- Out of the 72 received, 24 were managers or had managerial responsibilities

- 68 out of the 72 respondents have been directly affected by automation in one form or the other.

\section{Perceptions}

The survey indicated most employees with Bachelor's degrees and above had positive perceptions of automation, while most older and less educated individuals had negative perception. Those who spoke negatively, in my opinion, are usually afraid of losing their jobs to machines. Others who were in favor and spoke positively about automation had their concerns as well. They felt automation of spacecraft operations could or would make their jobs boring and less challenging. They were also concerned that the analysts or operators might lose their skills and become less alert if some of their tasks were automated. Both of these reasons, according to the respondents, could result in job dissatisfaction and quitting or moving on to other jobs that provide more challenge.

Majority also felt that taking the human element out of spacecraft operations should be minimized. The reason commonly given on the questionnaire was that automation, even though a good idea on some projects or missions, could have some flight operations risks associated with it. In other words, people might not know what to do when automation fails. In the airline industry, pilots have a high-level situational awareness even when the auto-pilot is activated. This analogy might apply to spacecraft operations in the sense that more tasks should be added to replace repetitive tasks that are being or have been automated. In doing so, job satisfaction could be enhanced while at the same time keeping interest in the job, especially on night shifts when human interaction is less common or non-existence. Among the people surveyed, $65 \%$ said they would start looking for new jobs when they realize their tasks were going to be automated.

Some of the positive responses were that automation would reduce work load, free up more time for analysis and contingency training, eliminate repetitive tasks, improve spacecraft monitoring, and most of all, would allow people to learn other things that might help improve their job performance. On a scale of 1 to 5 , automation was given an average of 3.5; but this should not be interpreted as a general approval for automation since the majority of the people who responded to the surveyed had bachelor's or higher degrees. This

U.S. Government work not protected by U.S. Copyright. 
group tends to support the implementation of automation.

The study brought an important and often overlooked subject to light, and that is employee morale. Employee morale plays a major role in cost reduction and savings. When morale is high, risks (including those related to human beings) are reduced and employee involvement goes on the rise.

According to the survey, employees' direct involvement in any automation project from the onset can help reduce cost. At a power generation plant visited, for instance, employees were asked to anonymously submit a list of areas that they thought needed automation. The feeling that it was not handed down from upper management increased participation and acceptance. This task increased morale, made the employees feel responsible for the success of the automation, and since they were more familiar with the actual work at the plant, they knew areas that might benefit the most automation.

The employees were empowered to make their suggestions heard even though the final decisions rested with management. The anonymous method ensured the elimination of the fear of retribution. It could be argued that, management could use this as a tool for eliminating jobs but the system was quite fair. Employee morale and input should therefore be taken into account when any automation is being considered in space flight operations. Their involvement gives some sense of pride, recognition and participation, which translates to enhanced job satisfaction and high morale.

\section{Implications}

The survey showed that while automation may be implemented for various reasons, the common factor was to make repetitive tasks easier and more accurate while reducing cost. The motivating reasons to automate ranged from budget cuts to modernization. According to the responses received from the power industries, automation was incorporated to speed up response times and to reduce "down-times" for the plants. The airlines, on the other hand, use auto-pilots modes to give their pilots some rest, while the automobile industries use automation to do precision and reliable jobs. No matter the reason, it could be concluded that automation has been very helpful in these industries and for that reason, automation should be considered in all present and future projects in order to improve spacecraft operations. This can be accomplished, as suggested earlier, by involving the flight operations teams in the automation process from the start.

Most people do not want to think about their jobs when they are home, but automation might make this impossible. Controllers, operators or spacecraft engineers, depending on the project, might be required to carry pagers around the clock seven days a week in order to respond to anomalies that might occur while the spacecraft is flown by automated procedures or programs onboard. Anomaly response time could be significantly affected by automation if "lights-out operations" is part or a result of the automation. Unless anomaly corrective measures are incorporated into the flight software of a spacecraft, this could be very detrimental to its health and safety. In the airline industry, for instance, the luxury of having a pilot override the auto-pilot exists. Automation, according to the survey will help in down sizing, but this was not the goal in the airline industry. This was, regrettably, one of the goals of car makers and the power industry.

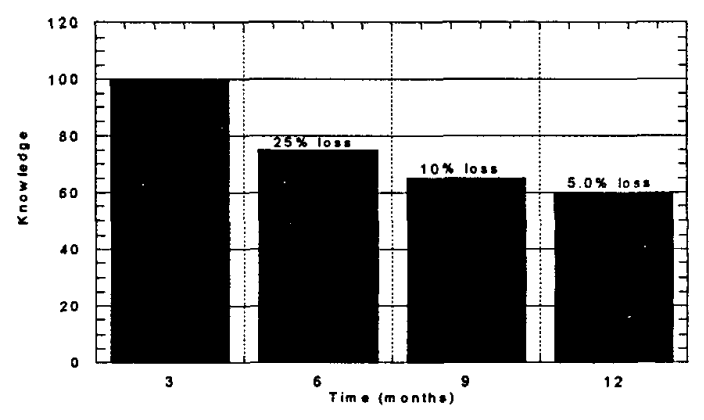

Automation could contribute to some new problems, including "knowledge atrophy", loss of expertise, and attrition. Atrophy usually sets in as people use their knowledge and skills less frequently, and tend to lose some of the knowledge base that had been acquired. The survey revealed that $25 \%$ of employees forget the manual method or way of doing things in three months, $35 \%$ in six months, and about $40 \%$ in a year. The conclusion can be drawn that as the years rise in number so does the level of atrophy. The loss of this knowledge cannot always be reversed, and could become detrimental to the health and safety of a 
spacecraft that experiences problems that needed manual intervention. In the airline industry, pilots get the opportunity to practice or use their skills when the auto-pilot is disabled but in spacecraft operations that could be a problem. A need would therefore exist to have people occasionally perform automated tasks manually to avoid knowledge atrophy from setting in.

Loss of experienced personnel is another serious problem that automation creates. From the survey, most people would start looking for other jobs as automation is being incorporated or considered. This could lead to experts or knowledgeable individuals leaving the team or project. For knowledge atrophy and personnel loss, a good documentation, knowledge management system and training process should be rigorously employed to minimize the impact. Downsizing can be a problem too, and should only be executed after successful implementation of automation; it should never be done before.

Some of the respondents raised legitimate concerns about the automation of existing systems. Responses from the flight operations teams, power, and the automobile industries, made it clear that automating existing operations sometimes created more problems than they were intended to solve. About 70 percent of those who replied to the survey said they would avoid incorporating automation into an old or existing system if they had a second chance or say in its implementation. Many believed

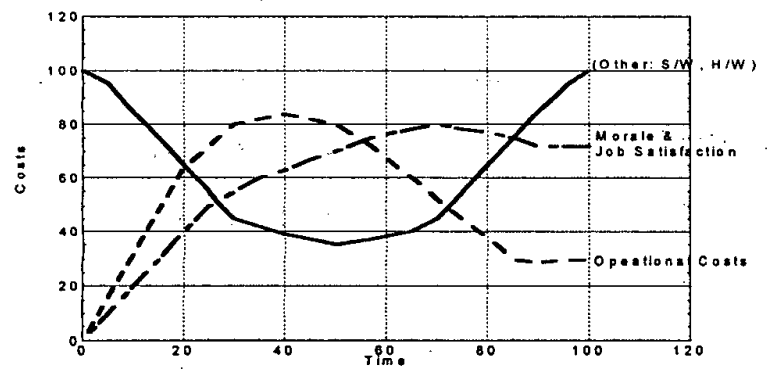

that automation implementation should by large be limited to new projects or systems and all efforts should be made to minimize its incorporation into existing ones. A graphical representation is shown above. Their concerns are well founded, since a major change in any requirement of a system will most likely create or generate known/unknown risks and problems.

\section{Cost Savings}

Almost all of the managers who responded said automation reduced operational cost. However, 80 percent said it increased other costs, including software development, installation, equipment, training, and even employee separation benefits. Some concluded that on some of the existing projects, automation cost savings were artificial and only "robbed Peter to pay Paul". A thorough cost/benefits analysis should therefore be done before any existing task and/or system is automated.

Making automation part of new projects was very much encouraged by all. In the spacecraft industry, it costs millions of dollars to place and maintain a spacecraft in orbit. Even though automation might save operations cost, it could jeopardize some missions, so care must be taken as to what really needs to be automated and what must not be automated. One major failure due to automation in some cases could wipe out the cost savings that were accomplished.

\section{Human Factors}

All the industries that were polled agreed that there was some job loss or re-assignments that can be directly attributed automation. Most of those who lost their jobs to automation were, however, reassigned. According to the survey, 90 percent remained employed within their organizations even though they were not doing what they did before the automation. In the automotive industry, all efforts were made to re-assign displaced workers within other units of their organization. Even though it did not erase the perception entirely, it helped employee morale and drastically reduced the fear of job loss, which is unfortunately sometimes associated with automation.

The survey also tried to determine how employees who have some experience with automation in one form or the other felt about it, before and after it was implemented. According to the survey, based on a rating scale of 1 to 10 , employee morale dropped from 9.5 to 6.5 after automation. This was across all industries surveyed, with the exception of the airline industry. For 
whichever reason, employee morale dropped considerably and this calls for further investigations. One may speculate that job loss, boredom and failure or other problems created by automation may take some blame for the drastic drop in morale. The diagram on the previous page show the correlation between morale, knowledge atrophy and cost.

Training and education should be made an integral part of automation implementation process if a decrease in employee morale is to be minimized. Training of those whose jobs might be affected is highly recommended. Improving the skills of those who will be working with the automated system will not only increase morale, but also enhance performance and satisfaction, according to the responses.

\section{Conclusion}

Automation could be used as a cost reduction or system improvement tool but all efforts must be made to ensure the health and safety the spacecraft and the mission as a whole. Automation has saved the automotive industry millions of dollars and it can do the same to the spacecraft industry in this era of budget cuts.

Manual ways of doing things should not be completely discarded. If possible, the various missions should try to develop a system that would enable some of the controllers or operators to perform manual functions on the project periodically. Manual redundancies or overrides should be built into all automated processes. Airplanes have manual redundancies that are used in disengaging the auto-pilot when it malfunctions. Employees should be trained to handle both manual and automation processes just like pilots - who can fly an airplane on auto pilot and manually. Automation on older and/or existing missions should only be encouraged after a thorough risk and cost/benefits analysis has been done, recognizing that automation, like all operational changes, involves some risks.

In February 2000, EUVE spacecraft experienced a problem, which could have caused the mission. It was reported that "...the controller loaded an old (incorrect) version of the HGA gimbal table, and (2) later that night the on-call engineer did not properly respond to the automated paging alarm from the ground systems that indicated empty-table track mode". This clearly implies that humans beings have a role to play when it comes spacecraft operations no matter how advanced technology might be or highly automated the systems is, so various human factors have to be taken into account when spacecraft operations are being automated.

When the problems and/or issues discussed here are properly understood and resolved, the risks associated with automation can be mitigated. The airline, automotive, and power industries have done it, and so can the spacecraft industry.

\section{Acknowledgment}

The greater part of this work was completed at NASA Goddard Space Flight Center where I worked during the research. I would like to thank Mr. Steve Tompkins of NASA/Goddard Space Flight Center in Greenbelt, Maryland, for approving the grant for this project; this project would not have been possible without such a generous grant from NASA. I would also like to thank Mr. Evan Eller of Honeywell Technology Solutions, Inc. for his interest in this project. I would like to thank my former manager, Mr. Lou Kurzmiller for giving me the opportunity and time to work on this project while working under him. My thanks also go to Mr. Andy Calloway of Honeywell Inc., who painstakingly edited the original draft. My gratitude also goes to present colleagues and former colleagues, whose views helped shaped this paper. I would like to thank my wife, Monica Kludze, who helped me in tabulating the survey. Finally, I would like to express my profound gratitude and appreciation to NASA Langley Research Center, especially my current boss, Mrs. Sharon Crockett, Head of the Systems Engineering Office/Systems Engineering Competency for making it possible for me to publish and present this paper. 


\section{References:}

Logsdon, Tom 1995. Mobile Communications Satellite: Theory and Application. New York:

McGraw Hill.

Zafanella, Carlo. 1999. "Reducing Cost and Increasing Volume by a Factor of 10 in the Space Component Business." Journal of Reducing Space Mission Cost, Vol.1 no.2

Boden, Daryl G. and Wiley J. Larson. 1996. Cost Effective Space Missions Operations. New York: McGraw Hill.
Wertz James R. and Wiley J. Larson. 1999. Space Mission Analysis and Design. Microcosm Press, California.

Sedano, Robert J. 2000. EUVE Incident Report- 23 February 2000. Goddard Space Flight Center, Maryland. 